
allemande

45-2 | 2013

Images et discours de la nation

\title{
Nationale Mythen und avantgardistischer Widerstand bei Christoph Schlingensief
}

\section{Franziska Schößler}

\section{(2) OpenEdition}

1 Journals

Édition électronique

URL : https://journals.openedition.org/allemagne/1489

DOI : 10.4000/allemagne.1489

ISSN : 2605-7913

Éditeur

Société d'études allemandes

Édition imprimée

Date de publication : 30 décembre 2013

Pagination : 297-307

ISSN : 0035-0974

\section{Référence électronique}

Franziska Schößler, „Nationale Mythen und avantgardistischer Widerstand bei Christoph

Schlingensief", Revue d'Allemagne et des pays de langue allemande [Online], 45-2 | 2013, Online erschienen am: 29 Juli 2019, abgerufen am 18 Mai 2021. URL: http://journals.openedition.org/ allemagne/1489 ; DOI: https://doi.org/10.4000/allemagne.1489 


\title{
Nationale Mythen und avantgardistischer Widerstand bei Christoph Schlingensief
}

\author{
- Franziska Schößler*
}

Schlingensief hat sich wiederholt mit Ikonen und Mythen der deutschen Geschichte auseinandergesetzt, allem voran mit dem Nationalsozialismus. „Immer wieder kehrt Schlingensief an die Wurzeln des deutschen Faschismus zurück. Es geht dabei darum, die großen Inszenierungen des Faschismus (und seiner Bearbeitung) zu unterlaufen, die Vertreter auf ein normales, eben barbarisches Maß zurückzuschrauben “ ${ }^{(1)}$. Er behandelt den Nationalsozialismus dabei nicht als historisches und damit vergangenes Phänomen einer linearen (Fortschritts-)Geschichte, sondern „[he] frames fascism as retaining a transhistorical and transnational potential well after Hitler's demise “(2). Insbesondere Schlingensiefs intermediales Verfahren fragmentiert und hybridisiert Geschichte, die individuelle wie die kollektive, und setzt so Verdrängtes frei. In diese heterogene Trümmerlandschaft fügt der Künstler avantgardistische Widerstandsformen gegen die gewaltvolle Geschichte ein: den Wiener Aktionismus, der gegen die österreichische Variante der Verdrängung gerichtet ist ${ }^{(3)}$, Joseph Beuys' Aktionen, Fassbinders Antitheater und anderes mehr. Fokus dieser Widerstandsformen ist der Körper als Medium des Widerstands (gegen nationale Mythen und geschlossene ästhetische Oberflächen), wie die Materialaktionen der Wiener Aktionisten auf besondere Weise verdeutlichen. „Der

* Prof. Dr., Universität Trier.

1 Georg Seesslen, „Vom barbarischen Film zur nomadischen Politik“, in: Julia Lochte, Wilfried Schulz (Hg.), Schlingensief! Notruffür Deutschland!, Hamburg, Rotbuch Verlag, 1998, S. 40-78, hier S. 52.

2 Richard Langston, „Schlingensief’s Peep-Show. Post-Cinematic Spectacles and the Public Space of History“, in: Randall Halle, Reinhild Steingrover (Hg.), After the Avant-garde: Contemporary German and Austrian experimental film, Rochester, Camden House Inc., 2008, S. 204-223, hier S. 206. Die Wiederkehr der untoten Vergangenheit inskribiert Schlingensief zudem seiner magischen Autobiographie, wie es Georg Seeßlen genannt hat; G. SEEssLen, „Vom barbarischen Film zur nomadischen Politik“ (Anm. 1), S. 69f.

3 Vgl. dazu auch Peter Turrini, Danièle Roussel, Der Wiener Aktionismus und die Österreicher, Klagenfurt, Ritter, 1996, S. 94. 
(eigene) Körper wurde politisiert und als Waffe gegen den Staat verwendet“ (4), unter anderem durch eine Körpermalerei aus Fleisch und Blut ${ }^{(5)}$.

Schlingensiefs hybrides, grenzauflösendes Bildprogramm, das sich jeder ordnungsstiftenden Rahmung entzieht, lässt sich deshalb als Angriff auf das identitäre Subjekt (samt seiner Meisternarrative wie das der Nation) beschreiben ${ }^{(6)}$. Es durchbricht die stabilisierenden Kopplungen von Identität, autonomem Subjekt und Nation ${ }^{(7)}$ und versucht immunisierende „Schutzdichtungen“ zu zerschlagen. Diesen Begriff Sigmund Freuds greift Elisabeth Bronfen in ihrer Analyse der Nation auf: Die imaginäre Erzählung des Nationalen ${ }^{(8)}$ ermögliche es, den verunsichernden Bedingungen der Moderne - dem Zufall, der Bedeutungslosigkeit und der Anonymität - zu entkommen. Die Nation als Vision einer Gemeinschaft stelle Sinn und Kohärenz her, der Bronfen „das verknotete Subjekt“ entgegensetzt ${ }^{(9)}$, Schlingensief den ästhetischen Bruch, das fragmentarisierte Bild, die Wunde und die Reise in den persönlichen Terror. Der Wiener Aktionismus entwarf entsprechend Versuchsanordnungen wie die Blutorgel (1962), bei der es darum ging, in die eigene Psyche hinabzusteigen, die durch einen Keller als „Brutanstalt“ konkretisiert wurde ${ }^{(10)}$.

Schlingensiefs Euvre lässt sich als serielles Bildprogramm diaphanischer Überlagerungen beschreiben, das im Anschluss an Avantgarde-Regisseure wie Kurt Kren und Alexander Kluge auf die Verstörung der Zuschauer/innen zielt. Seine Überblendungen fungieren als fragmentierte Spiegel, die keine geschlossenen Oberflächen präsentieren, sondern blinde Flecke in das Spiegelbild (der Zuschauer/innen) eintragen. Schlingensiefs Bilder perforieren im Sinne der Breton'schen Definition des surrealistischen Kunstwerks - dieses bestehe darin, in die Masse zu schießen - die Identitätskonstruktionen der Zuschauer/innen, lassen die Grenze zwischen Subjekt und Abjekt (dem Müll, dem Ausgegrenzten, der Ausscheidung) kollabieren und enthüllen die menschliche Verwundbarkeit (als Sterblichkeit). Sie setzen die Reise ins Innere („ins Schwein“) und das eigene Unheimliche, „das Fremde in mir“, gegen die schönen Oberflächen und Identitätsfiktionen, die

4 Brigitte Marschall, Politisches Theater nach 1950, Wien/Köln/Weimar, UTB/Böhlau, 2010, S. 401.

5 Ebd., S. 402.

6 Langston zieht Parallelen zu Alexander Kluges filmischem Werk, das ebenfalls durch Überlagerungen und andere Avantgarde-Techniken versucht, „unsichtbare Bilder“ zur Aktivierung der Zuschauer/ innen freizusetzen; R. LANGSToN, „Schlingensief's Peep-Show“ (Anm. 2), S. 208.

7 Diese bedarf stellvertretender, imaginärer, heroischer Subjekte (wie Siegfried, Barbarossa, Wilhelm Tell oder Adolf Hitler); so betont Hinrich C. SeEbA, „Fabelhafte Einheit. Von deutschen Mythen und nationaler Identität“, in: Claudia MAy ER-Iswandy (Hg.), Zwischen Traum und Trauma - Die Nation. Transatlantische Perspektiven zur Geschichte eines Problems, Tübingen, Stauffenburg, 1994, S. 59-74, hier S. 59. 1989 wird verblüffenderweise in den Zeitungen noch einmal der Barbarossa-Mythos aufgerufen.

8 Vgl. Benedict Anderson, „Kulturelle Wurzeln“, in: Elisabeth Bronfen, Benjamin Marius, Therese StefFen (Hg.), Hybride Kulturen. Beiträge zur anglo-amerikanischen Multikulturalismusdebatte, Tübingen, Stauffenburg, 1997, S. 31-58.

9 Vgl. Elisabeth Bronfen, „Einführung“, in: Ebd., S. 1-19, hier S. 2 f.

10 Otto Mühl formulierte: „Unter einem Künstler verstehe ich einen Menschen, der sich unter die Erde begibt, dort Stollen treibt, und solange alles kreuz und quer unterminiert bis plötzlich irgendwo sich ein Erdrutsch ergibt“; zitiert nach Dieter SchwArz, Aktionsmalerei, Aktionismus - Wien, 1960-1965, Zürich, Seedorn, 1988, S. 41. In dieser Äußerung zeigt sich die Koinzidenz von Widerstand und Freud'schem Ich-Modell, dessen Es bekanntlich im Keller wohnt. 
den Menschen regierbar machen - deshalb ist sein hybrides Bildprogramm Bestandteil eines politischen Projekts, das die Angst im Inneren (als Bedingung von Autonomie) sucht, nicht aber im Außen (als Voraussetzung von Regierbarkeit).

Die „Bildstörungsmaschinen“ seiner Filme und Theaterarbeiten deformieren allem voran die, heroischen 'Bilder des Faschismus, dessen untote Gespenster über die Wiedervereinigung hinaus (nicht nur in seinen Produktionen) ihr Unwesen treiben. Schlingensief führt aus, dass man Hitler „[e]ssen und benutzen“ müsse ${ }^{(11)}$, bzw. dass Hitler aufgrund seiner ungebrochenen Ikonisierung nach 1945 nicht ausreichend abgenutzt worden sei. Die Obsessionen des Künstlers für Splatter, den Körper im Schmerz, für Folter und Lust ${ }^{(12)}$ versuchen diese Mythenbildungen zu unterbrechen - Schlingensiefs Film 100 Jahre Adolf Hitler. Die letzte Stunde im Führerbunker beispielsweise lässt sich als (vorgezogener) Kommentar auf Eichingers Der Untergang lesen ${ }^{(13)}$, der in der europäischen Tradition des SS-Sexploitation ${ }^{(14)}$ Macht, Gewalt und sexuellen Exzess zusammenführt und dessen Trash-Ästhetik ebenso gegen Hollywood wie gegen nationale Ikonen gerichtet ist.

\section{Nation als Exklusion und Fiktion in Ausländer raus. Bitte liebt Österreich}

Dass für Schlingensiefs Versuchsanordnungen die Nation (in ihrem konstitutiven Zusammenhang mit Identität) eine entscheidende Rolle spielt, zeigt sich nicht zuletzt daran, dass er seine spektakulären Aktionen bevorzugt in anderen Ländern durchführt, Ausländer raus in Österreich, sein „Resozialisierungsprojekt“ Nazis rein in der Schweiz ${ }^{(15)}$. Er nutzt mithin die Sprengkraft nationaler Grenzziehungen und ihre verknappenden Diskurse: Die konservative Schweizer Partei SVP um Christoph Blocher reagiert auf Schlingensiefs Hamlet-Projekt, das eine Aussteigerkampagne der deutschen Bundesregierung kopiert und ironisiert, mit der Anfrage, ob sich dieses Experiment am Zürcher Schauspielhaus nicht verbieten lasse. Prompt fordert Schlingensief, diese Geste parierend und vergrößernd, die SVP zu verbieten, was helle Empörung auslöst und den notorischen Nationaldiskurs regelrecht hysterisiert: Wie könne sich ein Deutscher erlauben, eine Schweizer Partei verbieten zu wollen; wie aber, so kontert

11 Zitiert nach Roman Berka, Christoph Schlingensiefs Animatograph. Zum Raum wird hier die Zeit, Wien/New York, Springer, 2011, S. 144.

12 Kris Thomas-Vander Lugt, „Better Living through Splatter. Christoph Schlingensief’s Unsightly Bodies and the Politics of Gore“, in: Steffen Hantke (Hg.), Caligari's Heirs: The German Cinema of Fear after 1945, Lanham, Md. [u.a.], Scarecrow Press, 2007, S. 163-184, hier S. 164.

13 So Burkhardt Lindner, „Schlingensiefs Untergang“, in: Margit Frölich, Christian Schneider, Karsten Visarius (Hg.), Das Böse im Blick. Die Gegenwart des Nationalsozialismus im Film, München, text und kritik, 2007, S. 98-110. Obszönität dient der Entzauberung der „Großen“; ebd., S. 103.

14 Vgl. K. Lugt, „Better Living through Splatter“ (Anm. 12), S. 168.

15 In Nazis rein, dem Züricher Projekt, das von Peter Kern dokumentiert wird, iteriert und konfundiert Schlingensief die binäre Ordnung von Aus- und Einschluss durch ihre Theatralisierung. Er schließt seine Aufführung an ein Projekt für ausstiegswillige Neonazis an, die vielfach aus dem kommunikativen Raum der Mehrheitsgesellschaft total exkludiert sind. Durch seinen Transfer der Aktion in die Schweiz - Nazis rein - verweist er zugleich auf die Präsenz des Antisemitismus in diesem Land. Die Inklusionsgeste verweist mithin auf die Exklusionsstrategien der Schweizer Nation, die bis zur Leugnung dieses Phänomens gehen. Zugleich bedeutet der Ausstieg der Neonazis einen Einsteig in die kapitalistische Gesellschaft der Dramaturg Carl Hegemann weist darauf hin, dass aus dem Neonazi dann eben ein Banker werde. 
Christoph Marthaler, der Schlingensief nach Zürich eingeladen hatte, könne einem Deutschen verboten werden, eine Schweizer Partei verbieten zu wollen. Zum Kalkül Schlingensiefs gehört also die künstlerische Arbeit in diversen Ländern, um den trotz Globalisierung weiterhin virulenten Nationaldiskurs zu mobilisieren ${ }^{(16)}$.

Schlingensief führt in seinen partizipativen Projekten die (imaginären) Identitätskonstruktionen der Nation zudem als Verfahren der In- und Exklusion vor und thematisiert die nationalen Grenzen samt der dominanten Herrschaftsform, also der scheinbar allinkludierenden Demokratie. In seinem Beitrag für die Wiener Festwochen, Ausländer raus, fusioniert Schlingensief Theater, Fernsehen, Internet und Politik deshalb hinsichtlich ihrer Selektionspraktiken. Basiert die Demokratie auf einem Wahlverfahren, das die repräsentative Stellvertretung legitimiert, so kann Selektion auch die Grenze zwischen nationalem Innen- und Außenraum herstellen und Menschen eliminieren. Das demokratische Procedere erscheint auf diese Weise als Verfahren, das Existenzberechtigungen verteilt, bestimmte Interessen repräsentiert, andere hingegen ignoriert und Personen auch ganz buchstäblich zum Verschwinden bringen kann.

Schlingensief entwickelt, um diese Selektionen bzw. Exklusionen erfahrbar zu machen und in die Körper einzuschreiben, eine „Bildstörungsmaschine“, die die ikonischen Repräsentationen der „imagined community“ Österreich - ein Zentralbegriff von Benedict Anderson - unterbricht. Diese ,Maschine‘ nimmt Verkörperungen und Visualisierungen vor, um die abstrakte politische Rede (beispielsweise den Slogan "Ausländer raus") in liminalen Bildern zu konkretisieren ${ }^{(17)}$, ungenießbar zu machen und die Evidenzeffekte des Visuellen ${ }^{(18)}$ zu stören: „Bilder zu allem, was schon lange ansteht und sich breitmacht“, so lautet das Programm. Die Visualisierung nimmt die politische Sprache beim Wort, zertrümmert ihre Metaphern und verweist auf die Auslassungen der politischen Bildpraxis. Schlingensiefs Installation Ausländer raus generiert Bilder, die im Sinne von Roland Barthes ein „punctum“ besitzen, das heißt Störung, Unreinheit sowie Heterogenität produzieren ${ }^{(19)}$ und verletzen können - das „punctum“ schießt „wie ein Pfeil aus seinem Zusammenhang hervor, um mich zu durchbohren“(20) und produziert ein „Mehr an Sichtbarem“(21). Die Versuchsanordnung, der Einschluss Fremder in Lagern, die an den (Austro-)Faschismus erinnern, lässt die Teilnehmer/innen auf dem Opernplatz tatsächlich zuweilen unter Schmerzen zusammenbrechen, wie der Film von Paul Poet dokumentiert - Schlingensief erinnert in diesem Zusammenhang ausdrücklich an den Surrealisten André Breton.

16 Vgl. dazu Thekla Heineke, Sandra Umathum (Hg.), Christoph Schlingensiefs Nazis rein. Torsten Lemmer in Nazis raus, Frankfurt am Main, Suhrkamp, 2002.

17 Vgl. dazu Franziska SchößLER, „Wahlverwandtschaften: Der Surrealismus und die politischen Aktionen von Christoph Schlingensief“, in: Ingrid Gilcher-Holtey, Dorothea Kraus, Franziska SchößLER (Hg.), Politisches Theater nach 1968. Regie, Dramatik und Organisation, Frankfurt am Main/New York, Campus Verlag (Historische Politikforschung, Bd. 8), 2006, S. 269-293.

18 Vgl. dazu Andreas Beyer, Markus Lohoff, „Bildhandeln. Eine Einführung“, in: Dies. (Hg.), Bild und Erkenntnis. Formen und Funktionen des Bildes in Wissenschaft und Technik, Aachen/München/ Berlin, Deutscher Kunstverlag, 2006, S. 12.

19 Roland Barthes, Die helle Kammer. Bemerkungen zur Photographie, Frankfurt am Main, Suhrkamp, 1985, S. 31.

20 Ebd., S. 35.

21 Ebd., S. 53. 
Als Schlingensief das provokante Schild Ausländer raus auf dem Container enthüllt, fordert er die Touristen (als gewünschte Fremde) auf, zu fotografieren und die sichtbare Geste des Ausschlusses zu multiplizieren. Diese Fotografien sind subversiv und affirmativ zugleich, denn sie durchbrechen die Selbstdarstellung Österreichs und machen doch lediglich den politischen Konsens sichtbar. Die Installation lässt mithin aktivierende Bilder (der Verletzung) entstehen; nach Barthes garantiert das "punctum“ die Beteiligung des Betrachters: „die Lektüre des punctum (des ,getroffenen' Photos, wenn man so sagen kann) ist [...] kurz und aktiv zugleich, geduckt wie ein Raubtier vor dem Sprung“ (22). Schlingensiefs liminale Bilder sind Orte, an denen die Systeme zu tanzen beginnen, weil sich die Kraftfelder gegenseitig stören und sich eindeutige Rahmungen auflösen ${ }^{(23)}$.

In einem Interview bezeichnet Schlingensief den Container zudem als Medium, das aus der Distanz heraustritt und die Zuschauer/innen in ihren eigenen Film integriert. Der Container auf dem Wiener Opernplatz sei eigentlich leer und fungiere als Screen, der kollektive Befindlichkeiten sichtbar mache, wie Schlingensief in einem turbulenten Interview im ORF am 13.6.2000 erklärt: „Das, was wir machen, ist eine Selbstprovokation - eine leere Fläche, auf die projizieren Sie Ihr Bild drauf - Ihren Film -, und Sie haben pausenlos das Problem, dass sich die Bilder gegen Sie selbst kehren “ (24). Die Akteure auf dem Wiener Opernplatz produzieren mithin ihren eigenen (verletzenden) Film über Traumata. Die Verstörung der Teilnehmenden ist Resultat ihres eigenen Fremden, ihrer Reise in die Abgründe des Selbst und die verdrängte Vergangenheit, zu den untoten Gespenstern des Nationalsozialismus, die Schlingensief (auch als Teil seiner magischen Autobiographie) exorzistisch beschwört ${ }^{(25)}$.

\section{Der Wiener Aktionismus in Attabambi-Pornoland}

Schlingensief hat sich für diese ,Reisen ins Innere` wiederholt auf die Materialaktionen der Wiener Aktionisten bezogen, beispielsweise in Kühnen 94 und in der Bambiland-Serie ${ }^{(26)}$. Er rearrangiert die gewaltvollen avantgardistischen Happenings einer

22 Ebd., S. 59.

23 Vgl. zum Konzept der Rahmung, das unsere Wahrnehmung reguliert und unter anderem die Unterscheidung zwischen schützenswertem und nicht-schützenswertem Leben entstehen lässt, Judith BUTLER, Raster des Krieges. Warum wir nicht jedes Leid beklagen, Frankfurt am Main, Campus Verlag, 2010. Die fluiden Bilder Schlingensiefs versuchen diesen Rahmungen zu entgehen.

24 Schlingensiefs Ausländer raus. Bitte liebt Österreich, Dokumentation von Matthias LiLIENTHAL und Claus Philipp, Franfurt am Main, Suhrkamp, 2000, S. 100. Sein Beitrag für die Wiener Festwochen führt die Reality-Show Big Brother, die den Voyeurismus des Fernsehens zum Selektionsspiel potenziert, mit dem öffentlichkeitswirksamen Thema der Ausländerpolitik zusammen. Per Internet können Asylbewerber (scheinbar) abgeschoben werden; sie befinden sich in einem Container, einem Lager - nach Giorgio Agamben eine unhintergehbare Begleiterscheinung westlicher Demokratien und ihrer Biopolitik, die Menschen als reines Leben behandelt und ,unwertes' aussondert. Schlingensief treibt also die Allianz von Demokratie und nationalem Ausschluss performativ hervor und macht die Beobachter zu Mittätern, so dass sie die Implikationen ihrer politischen Forderungen am eigenen Leibe spüren. G. Seesslen, „Vom barbarischen Film zur nomadischen Politik“ (Anm. 1), S. 69f. Brus und Kurt Kren gewidmet ist und sich auf die berühmte Aktion Selbstverstümmelung bezieht; vgl. R. Berka, Christoph Schlingensiefs Animatograph (Anm. 11), S. 336. 
Gruppe, die sich dezidiert gegen die Verdrängung des Austrofaschismus richtet und das Ausgegrenzte, den Müll, die „Versumpfung“ des Körpers und die Kastration (als Angriff auf die Ikone der Macht) in Szene setzt. Im Anschluss an ein psychoanalytisches Modell sollen die individuelle Sublimation sowie die staatliche Repression durch den Exzess überwunden werden. Insbesondere mit Günter Brus und Rudolf Schwarzkogler, deren Aktionen noch in Mea Culpa als Readymades eingespielt werden, sind Künstler benannt, die Medien kombinieren und transformieren - stilllebenartige Kompositionen und pantomimische Filmsequenzen verfremden den Körper und zeigen ihn als verletzten, gefolterten - und auf diese Weise die Verdrängung des Faschismus in der österreichischen Gesellschaft der 1960er Jahre bearbeiten ${ }^{(27)}$. Politische Kunst ist mithin Körperarbeit, abstrakte Gewalt wird in die fluiden, ungerahmten Bilder schmerzender und verstümmelter Leiber übersetzt.

Der Wiener Aktionismus spielt auch in dem Zyklus Bambiland eine zentrale Rolle - Elfriede Jelineks Neologismus für die „Verplüschung“ der medialen Gesellschaft in der Zeit des Irakkrieges. Schlingensief inszeniert 2003 Atta Atta - Die Kunst ist ausgebrochen und Bambiland, Theaterabende, die sich in seriellen Selbstzitaten auf Jelineks Vorlage beziehen, allerdings eher als kongeniale Übersetzung zu bezeichnen sind. 2004 folgt die Produktion Attabambi-Pornoland, die Jelineks Monologe Irm sagt und Margit sagt aus Babel intermedial umspielt. Alle drei Abende, die auf die apokalyptischen Medienszenarien nach dem 11. September 2001 und die sich daran anschließenden Kunstdebatten (rund um Stockhausens Äußerung über die einstürzenden Twin Towers als Kunstwerk) reagieren, gehören zu dem am 20.3.2003 gegründeten Projekt „Church of Fear“", das den Terror im Ich sucht, nicht in der medialen Inszenierung von Bildern des Bösen. Gemeinsamer Gegner von Jelinek und Schlingensief scheint also das hypnotische (Medien-)Bild zu sein, das der künstlerische Akt zu zitieren und zerstören versucht. Inszeniert Schlingensief Bilder als Überschuss und Überwältigung des Logos, so unterbricht auch Jelinek deren Identitätslogik ${ }^{(28)}$ : Im Monolog Peter sagt reagiert der Sprecher mit einem Schrei, als er von seinem Bild abgetrennt wird und das Objekt seinem Wort nicht mehr entspricht - ein Vorgang, den hier der gewaltvolle MarsyasMythos illustriert ${ }^{(29)}$.

Attambambi-Pornoland, das mit geradezu wuchernden filmischen Übermalungen der gesamten Bühne arbeitet (sehr viel stärker als die vorangegangenen zwei Produktionen), besteht aus einem Set an Referenzen, die Schlingensief auf unterschiedlichen medialen Ebenen - Musik, Bild und Sprache - kombiniert. Eingespielt werden mit großer Regelmäßigkeit und im Vorgriff auf Schlingensiefs Bayreuth-Inszenierung Musik und Szenen aus dem Bühnenweihfestspiel Parsifal und der Götterdämmerung ${ }^{(30)}$.

27 Rosemarie Brucher, Durch seine Wunden sind wir geheilt. Selbstverletzung als stellvertretende Handlung in der Aktionskunst von Günter Brus, Wien, Löcker, 2008, S. 15.

28 An einer Stelle heißt es: „Nie spurlos fort unserer Gottheit Bilder, die wir dort sehn, die nur wir dort sehn auf dem leuchtenden Schirm. So, wir entfernen dieses Volk vom Glauben, geben ihm dafür endlich unser Bild und aus." Elfriede Jelinek, Bambiland/Babel. Zwei Theatertexte, Reinbek bei Hamburg, Rowohlt, 2004, S. 19.

29 Vgl. dazu Bärbel Lücke, „Zu Bambiland und Babel. Ein Essay“, in: Ebd., S. 239f.

30 In dem Monolog Irm sagt aus Babel, in dem Pornographie, Krieg und Religion als Arbeit am Körper verknüpft werden, wird die Seitenwunde Christi mit der von Amfortas in eins gesetzt und beide als 
Gleichzeitig wiederholen die projizierten Filme sowie die theatralen Vorgänge die Aktionen von Joseph Beuys und der Wiener Künstler/innen - Günter Brus, Hermann Nitsch und andere werden, wie schon in der Produktion Bambiland, explizit benannt; eingespielt werden zudem dadaistische Lautgedichte ${ }^{(31)}$. Die Schauspieler/innen sprechen kurze Textpartien aus Jelineks Babel, ebenfalls in Wiederholung. Der Abend folgt einer seriellen Kombinatorik, die Zeit- und Raumeinheiten auflöst und eine ,andere Zeit' spürbar werden lässt. Die Serie (als avantgardistisches Verfahren) stellt die bürgerlichen Mythen des Originals und einer kontinuierlichen Geschichte in Frage.

Jelineks Monologe umkreisen die Phallusidolatrie des Kriegs ${ }^{(32)}$, der Pornographie ${ }^{(33)}$ und der Religion und beschwören als ihre Kehrseite die Kastration(-sangst) ${ }^{(34)}$; Attabambi-Pornoland stellt entsprechend Körperaktionen der Wiener Künstler/innen nach, allem voran die auf das Genital konzentrierten Aktionen von Hermann Nitsch (wie die 8. Aktion vom 22. Januar 1965) ${ }^{(35)}$ und die Verstümmelungen von Rudolf Schwarzkogler - während der 6. Aktion beispielsweise ist Schwarzkogler mit Mullbinden bandagiert und Elektrodrähten verschnürt; sein Penis ist mit Farb- und Blutspuren bedeckt als Vorstufe der Kastration ${ }^{(36)}$. Durch die Filme in Attabambi-Pornoland geistert zudem eine am ganzen Körper bandagierte Figur, die aus Schwarzkoglers 3. Aktion (Sommer 1965) stammt ${ }^{(37)}$. Die so zitierte Aktionskunst stellt mit ihren Angriffen auf den integralen Körper und die Suche nach „Enthemmungsekstasen“ und „Abreaktionsereignissen“, nach Rausch und dem „Erleben dionysischer Triebdurchbrüche“ (38), die sich vielfach gegen den Phallus (als Ausdruck der symbolischen Ordnung) richten, die Deckgeschichten und Verdrängungen der österreichischen Nachkriegsgesellschaft in Frage. Diese Kunst lässt - auch das demonstriert Schlingensiefs Reenactment - durch

„Geschlechtslappen“ bezeichnet; E. Jelinek, Bambiland/Babel (Anm. 28), S. 93. Anders als bei Schlingensief verstehen die Texte Jelineks die Wunde, wie sie die Religion glorifiziert, jedoch als Bestandteil eines domestizierenden (Opfer-)Apparates, der die Mutter zur Mittäterin werden lässt. Scheint Schlingensief den religiös-ästhetischen Diskurs, der den Künstler zum Opfer und zur Christusfigur stilisiert, für sich durchaus in Anspruch zu nehmen und nicht nur zu demontieren, so ist das Motiv der Wunde (samt der Erlösungshoffnung, für die protoptypisch Wagner steht) für Jelinek Bestandteil eines patriarchalen Opferdiskurses.

31 Der Dadaismus kann als Antwort auf den Nationalismus im Ersten Weltkrieg verstanden werden, auf die Hochwertparolen, die junge Männer in den Krieg schickten.

32 In Bambiland feiert Jesus-Bush zum Schluss „eine Orgie des Macht-Fetisch Phallus, die in der Verschränkungsfigur von Technik, Religion und Sexualität kulminiert“; B. Lücke, „Zu Bambiland und

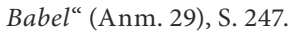

33 Sie legt den Zusammenhang von pornographischen Bildwelten („Titten und Mösen machen jetzt Geschichte“; E. Jelinek, Bambiland/Babel [Anm. 28], S. 90) und Gewalt bzw. Folter frei.

34 Jelinek überblendet in Margit sagt das Märchen von Hänsel und Gretel mit der religiösen Familiengeschichte, die zugleich eine kleinbürgerliche ist: Die Mutter als Märtyrermutter, die ihren Sohn durch die emotionalen Bindungskräfte und das inzestuöse Begehren in der Kleinfamilie zum Opfer vorbereitet, brät ihn im Ofen, um ihn sich wieder einzuverleiben. Die Kastration bzw. das ödipale Narrativ (hier konzentriert auf Mutter und Sohn) spielt dabei eine zentrale Rolle.

35 Vgl. Julius Hummel (Hg.), Wiener Aktionismus. Sammlung Hummel, Wien, Edizioni Mazzotta, 2004, S. $128 f$. 
die Gewalt gegen den Körper das Trauma der Folter und des Kriegs spürbar werden ${ }^{(39)}$. Avantgarde und Krieg treffen sich in der Aggression ${ }^{(40)}$.

Die Sound- und Bildprogramme überlagern die (Wiener) Aktionen mit WagnerKompositionen und umkreisen den Mythos von Telephos, vom Speer, der die Wunde wieder schließt, die er schlug (Parsifal). Auf der Leinwand sind wiederholt der bandagierte Schmerzensmann sowie ein blutender (bemalter) Körper zu sehen, der durch Schnitte, Nahaufnahmen, körnige Bildoberflächen und Einfärbungen in seine Bestandteile aufgelöst wird. Auch Schlingensiefs Kamera imitiert (wie die von Kurt Kren) das Action-Painting und zersetzt die Körper in abstrakte Bilder ${ }^{(41)}$. An anderer Stelle zeigen die Filmbilder eine auf den Penis zentrierte Aktion: Die zum Teil opulenten Körper werden durch Farben verändert - Brus verwendete umgekehrt Blut als Farbe - und eine Kastration inszeniert; zwei Beobachter (Carstensen und Schlingensief) lachen rauschhaft im Angesicht des geschundenen Körpers, worauf eine Kopulations- und Defäkationsszene folgt - eine der Vorlieben der Aktionisten, wie der berühmte Kurzfilm 16/1967 20. September auf drastische Weise verdeutlicht. Diese Bilder sind mit Zitaten aus Bambiland unterlegt, die davon sprechen, dass wir Gefühle nurmehr durch das Bambi als Ausdruck einer infantilisierten Bildwelt erreichen. Die fluiden, collagierten und überblendeten Filmbilder, die Pornographie und Schmerz, Lust und Folter engführen, konterkarieren diese Feststellung und lassen den Schock, das Unheimliche der aktionistischen Körperbearbeitung erfahrbar werden. Sie münden in eine Kamerafahrt in das Innere des Körpers, in den Schlund - der Weg geht nach Innen, in das „Schwein im Selbst“, das im Anschluss an die filmisch präsentierte Geburt eines Schweins die Bühne leibhaft betritt. Diese Bilder werden mit Wagners Götterdämmerung unterlegt, so dass Schlingensief auch hier die Sehnsucht nach Erlösung aufruft - die der Avantgarde wie die Richard Wagners ${ }^{(42)}$. Die Forschung hat den Bezug der Wiener Aktionisten zum Katholizismus hervorgehoben, wie er in Wagners theoretischen Schriften und Opern ebenfalls eine zentrale Rolle spielt. Schlingensief selbst bietet in Attabambi-Pornoland ironischerweise einen „Erste-Hilfe-Koffer der Erlösung“ an und erhebt die Pornographie zum Erlösungsprinzip.

39 Schlingensief verdeutlicht damit auch, was sich bereits in den Wiener Aktionen zeigt: dass die künstlerischen Ausdrucksformen diejenige Gewalt imitieren und vergegenwärtigen, gegen die sie gerichtet sind. Deshalb gleichen die künstlerischen Aktionen selbst gewaltvollen Übergriffen. Die filmisch und auch theatral präsentierten Remakes von avantgardistischen Happenings, die den Körper in seinem Schmerz und seiner dionysischen Auflösung (wie in den Versumpfungsaktionen) zeigen, erscheinen als Folter, wie sie Jelinek im dritten Teil von Babel zum Gegenstand macht: In dem Monolog Peter sagt geht es um die Vorfälle im Gefängnis von Abu Ghraib, um die Konnotation von Eros und Gewalt und die mediale Verwertung der Fotografien, die die Folterer gemacht haben.

40 Diese Koinzidenz diskutierte das Attaismus-Seminar; vgl. dazu Brechtje Beuker, „The Fusion and Confusion of Art and Terror(ism): Atta Atta“, in: Tara Forrest, Anna Teresa Scheer (Hg.), Christoph Schlingensief: Art without Borders, Bristol/Chicago, Intellekt, 2010, S. 137-151, hier S. 141.

41 Bei Kurt Kren sieht man zuweilen lediglich „farbige Materialien stauben, fallen, platzen, rinnen, kleben und die Körper, ihre Oberflächen und Teile, in ihrem Winden und ihren Verrenkungen, ihren Positionen und Gesten darin eingemengt“; Kren entwickelt ein Kino der Intensitäten und der Präsenz; Michael PAlm, „Which Way? Drei Pfade durchs Bildgebüsch von Kurt Kren“, in: Hans Scheugl (Hg.), Ex-Underground Kurt Kren. Seine Filme, Wien, PVS Verleger, 1996, S. 114-129, hier S. 116.

42 Erlösung sei unter anderem „das Stichwort für die therapeutische Funktion der, alten Fabeln`, der nationalen Mythen“, wie sie auch Wagner beschwört; H. C. SeEBA, „Fabelhafte Einheit“ (Anm. 7), S. 62. 
Die eingeblendeten Filme und die Aktionen auf der Bühne nehmen insgesamt eine Materialisierung des Körpers vor, wie sie auch die Wiener Aktionisten anstrebten, eine Entfunktionalisierung und Anverwandlung an die Dinge, die als Verweigerung an die normierte Waren- und Medienwelt sowie den Staat und seinen Funktionalismus ${ }^{(43)}$ verstanden werden kann. „Der materialisierte Mensch als das entzweckte, entmoralisierte, entzivilisierte, entpsychologisierte, entkodierte Wesen scheint der Repression über tradierte Bedeutungen und Ideen entgehen zu können “(44). Schlingensiefs intermediale Bühnenanordnungen generieren durch diese Materialisation (als Entmenschlichung) verletzende Bilder und perforieren die Grenzen zwischen Innen und Außen. Diese Bilder ziehen die Betrachter trotz ihrer visuellen Distanz in Mitleidenschaft, weil sie den ,ganzen Spiegel' verweigern - durch die Überblendungen, die ständige Bewegung, die Assoziationsfülle und Undeutlichkeiten. Ganz in diesem Sinne macht auch Splatter die Distanzierung unmöglich, „bringing the viewer into direct - and violent - confrontation with trauma, loss, and the returned repressed “(45). Die Bilder eröffnen Spielräume für ein projektives Sehen, das die verdrängten Triebe freisetzt. Schlingensief selbst beschreibt sein Bildprogramm bezeichnenderweise als „Schütteln“ der Zuschauer/innen: „Da muss man die Leute schütteln. Das Schütteln soll dazu führen, dass die Bilder im Kopf langsam frei werden, dass aber auch der Körper wieder freier wird“(46).

Die Aktionen der Wiener Avantgardisten werden in den Reenactments Schlingensiefs allem voran als Auseinandersetzung mit dem Nationaldiskurs deutlich, wie die regelmäßig eingeschalteten Parteitage profilieren. Schlingensief spricht auf der Bühne beispielsweise davon, dass er sich zur „Urwurzel des Deutschseins“ bekenne und prangert die Dialektalisierung in der Schweiz an - der Abend findet in Zürich statt. In einem Atemzug erwähnt er die Wiener Aktionisten Brus und Nitsch ${ }^{(47)}$, die auf die nationalen Flaggen defäkiert hätten - in dem Kurzfilm 16/1967 20. September werden die Ausscheidungsakte von Brus und Kren verlangsamt und in serieller Wiederholung gezeigt. Sie „scheißen buchstäblich auf Österreich und ein bürgerlich-snobistisches Kunstpublikum“ ${ }^{(48)}$, wobei die Demonstration des Abjekten auf die (staatlichen) Zurichtungen des Körpers und des Geistes verweist ${ }^{(49)}$.

43 Brus verklammert in seinen Äußerungen konsequent Staat und Körper: „Der Staat hält seine körperliche Hinfälligkeit dadurch aufrecht, daß er mich zur Krücke befiehlt. Ich soll für ihn denken, ihm seine Pflichten abnehmen, seinen Rotz wischen und Ausgleichssport für seine Gedärme üben. “Zitiert nach B. Marschall, Politisches Theater nach 1950 (Anm. 4), S. 116. Er anthropomorphisiert den Staat und führt ihn als Medium der Körperarbeit, -disziplinierung und Ausbeutung vor.

44 Kerstin Braun, Der Wiener Aktionismus: Positionen und Prinzipien, Wien/Köln/Weimar, Böhlau, 1999, S. 202.

45 K. LugT, „Better Living through Splatter “(Anm. 12), S. 166.

46 Zitiert nach Teresa Kovacs: „,60 Sekunden im Krieg‘. Christoph Schlingensiefs Umgang mit den Bildern des Irakkriegs in Elfriede Jelineks Bambiland“, in: Jelinek-Jahrbuch 2011, S. 207-219, hier S. 209.

47 Bei einem der weiteren Parteitage mutiert Schlingensief zu Hitler, imitiert den Hitler-Gruß und thematisiert das Verhältnis von Nazi-Deutschland und der Schweiz („Zürich soll knien“). An anderer Stelle lässt er eine Partie aus einer Oper von Carlos Gommes, dem bekanntesten brasilianischen Komponisten, nachspielen, und zwar eine Szene, in der es um das Vaterland und das Sterben dafür geht - doch einer der Sprecher erklärt das Vaterland für eine Anhäufung von Bergen und Flüssen.

48 M. PAlm, „Which Way?“ (Anm. 41), S. 114f.

49 In der Aktion Kunst und Revolution „schiß Brus auf den boden des hörsaals, verschmierte sich den scheißdreck am leib, stach mit seinen fingern den ösophagus hinab, würgte, erbrach, sang zum 
Was Schlingensief also gegen die Medienbilder der Angst, gegen die staatliche Repression und die Gespenster einer nationalen Vergangenheit setzt, ist eine Reise ins Innere („ins Schwein“), die sich durch die „hybriden Terrorikonen“ auf der Bühne gegen die apokalyptischen Inszenierungen von simplen Ordnungssystemen wendet. Das Theater selbst wird durch seine Bildfluten zum Krieg, die Zuschauer/innen mit Bildern, Musik und Texten bombardiert, die sich jedoch geschlossenen Oberflächen und rahmenden Sinnsystemen verweigern; die Instabilität und Unsicherheit des Terrorismus wird zur (ästhetischen) Erfahrung( ${ }^{(50)}$.

Doch der Künstler stellt den Wiener Aktionismus nicht nur nach, sondern unterzieht ihn (wie die avantgardistische Tradition insgesamt) einer kritisch-ironischen Revision, die der politisch-künstlerischen Analyse nach dem 11. September 2001 dient. Denn der Abend verdeutlicht (auch durch seine Jelinek-Bezüge), dass die fundamentale Geste, die den islamistischen Terrorismus so bedrohlich erscheinen lässt, die Opferung der Selbstmordattentäter und die Sehnsucht nach dem Paradies, einem westlichen Kunstmythos entspricht und in der Erlösungssehnsucht Wagners ebenso präsent ist wie in den auratisierenden Geniegesten der Avantgardisten. Stilisieren sich diese im Anschluss an die Romantik (die den Künstler zur Christusfigur und zum stellvertretend Leidenden stilisiert) zu Märtyrern, wird insbesondere Beuys die Selbstwahrnehmung als gottgleicher Künstler attestiert und unterstreicht Jelinek in dem Monolog Margit sagt den Opfergestus der christlichen Marienikone, so zeichnen sich westliche Kunst und Religion durch die gleiche Haltung der Opferung, des Märtyrertums und der Demonstration der Wunde aus, wie sie die Attentäter an den Tag legen. Günter Brus beispielsweise imitiert in seinen autoaggressiven Selbstverletzungen das christliche Opfer (um es zu überschreiten); er „verkörpert nackt, kahl rasiert und verletzt die christliche Metapher vom geschundenen Menschen“ (51).

Schlingensief hebt mithin den Clash of Cultures auf, indem das Fremde (die Opferung) als Vertrautes erscheint, und zwar in der avantgardistischen Kunst wie der christlichen Religion. Die Selbstauratisierung zum Opfer, die Materalisierung zum verwundeten Körper - als (verschobene) Imitation katholischer Bildwelten - entspricht dem Gestus der Selbstmordattentäter. Das Bildprogramm Schlingensiefs stellt die Grenzziehungen zwischen den Kulturen ebenso in Frage wie die zwischen Subjekt und Abjekt, Innen und Außen. Und sein Reenactment (der Wiener Aktionisten) dementiert eine lineare Geschichte, um so den eigenen (historischen) Ort zu markieren - erst das Zerbrechen historischer Kontinuität bringt die Gespenster zum Vorschein. Diese Entgrenzung versucht den ikonischen Kosmos wieder zu öffnen, der sich nach dem 11. September 2001 geschlossen hatte. Schlingensief hält über den Terrorangriff

scheißen die bundeshymne, onanierte“; Peter Weibel (Hg.) unter Mitarbeit von Valie Export, Bildkompendium wiener aktionismus und film, Frankfurt am Main, Kohlkunstverlag, 1970, S. 263. Er wurde daraufhin zu sechs Monaten Haft wegen Herabwürdigung von Staatssymbolen, der Ehe und des Eigentums verurteilt; B. Marschall, Politisches Theater nach 1950 (Anm. 4), S. 117. Schlingensief stellt zudem eine Frau seiner Gruppe vor, die mit Menstruationsblut arbeite - ganz ähnlich wie Nitsch damit düpiert hatte, dass er während der Aktion Erste heilige Kommunion Menstruationsblut mit Lammblut gemischt hatte.

50 B. Beuker, „The Fusion and Confusion of Art and Terror(ism)“ (Anm. 40), S. 147.

51 R. Brucher, Durch seine Wunden sind wir geheilt (Anm. 27), S. 83. 
fest, „dass die 3.000 bedauernswerten Toten in New York zu einer Paralysierung und zu einem Super-Flash geworden sind, der sämtliche andere Bilder verschüttet “ ${ }^{\text {(52) }}$.

Der Avantgardist Schlingensief zersetzt mithin die lineare Geschichte durch ein serielles intermediales Verfahren bzw. verletzende Bilder und zerstört auf diese Weise den Mythos historischer Linearität, der die Verdrängungen (des Faschismus) erst möglich macht. Er schichtet, einer Archäologie gleich, diverse Formate und Ereignisse übereinander, Machtgeschichte und Widerstandsformen, Nationalkriege und Terrorismus, Wagner (als nationale Ikone) und die Avantgarde und befreit durch sein diaphanisches Bildverfahren, durch das „Schütteln“ der Zuschauer/innen, die Gespenster der (nationalen) Geschichte aus den Kellern, um sie abzunutzen.

\section{Zusammenfassung}

Der Theater- und Medienkünstler Christoph Schlingensief setzt sich auf obsessive Weise mit der deutschen Geschichte und ihren neuralgischen Ikonen (wie Hitler) auseinander, die er, abzunutzen'versucht. Er entwickelt ein hybrides, grenzauflösendes Bildprogramm, das sich ordnungsstiftenden Rahmungen entzieht und Angriffe auf das identitäre Subjekt (samt seiner Meisternarrative wie das der Nation) lanciert. Schlingensiefs intermediales Verfahren fragmentiert Geschichte, setzt Verdrängtes frei und durchschlägt immunisierende „Schutzdichtungen“. In diese heterogene Trümmerlandschaft fügt der Künstler avantgardistische Widerstandsformen gegen die gewaltvolle Geschichte ein - beispielsweise den Wiener Aktionismus, der gegen die österreichische Variante der Verdrängung (nationalsozialistischer Geschichte) gerichtet ist, und Joseph Beuys' Aktionen. Schlingensiefs Projekte durchbrechen die stabilisierenden Kopplungen von Identität, autonomem Subjekt und Nation, wie an seiner kongenialen Adaption von Jelineks Bambiland, Attabambi- Pornoland, gezeigt werden soll.

\section{Résumé}

Christophe Schlingensief, metteur en scène et artiste multimédia, se confronte de manière obsessionnelle à l'histoire allemande et ses icônes névralgiques (telles qu'Hitler) qu'il cherche à "user " en les utilisant. Il élabore un programme visuel hybride qui transgresse les frontières entre les disciplines artistiques, se dérobe à tous les cadres structurants et lance ses attaques contre un sujet fondé sur une identité définie et ses " grands récits" tels que celui de la nation. Le procédé intermédial de Schlingensief fragmente l'histoire, libère le refoulé et transperce les " jointures " immunisantes. Dans ce paysage hétérogène de ruines, l'artiste insère des formes de résistance avant-gardistes à la violence de l'histoire tel que l'actionnisme viennois, qui est dirigé contre la variante autrichienne du refoulement de l'histoire nationale-socialiste, et les actions de Joseph Beuys. Les projets de l'artiste viennent rompre les corrélations stabilisantes entre identité, sujet autonome et nation, ce que fait apparaître l'analyse d'Attabambi-Pornoland, sa formidable adaptation de Bambiland d'Elfriede Jelinek.

52 Michael Kerbler, Claus Philipp, „,Ich bin für die Vielfalt zuständig. Christoph Schlingensief im Gespräch mit Michael Kerbler und Claus Philipp“, in: Christian Reder (Hg.), Lesebuch Projekte. Vorgriffe, Ausbrüche in die Ferne, Wien, Springer, 2006, S. 125-140, hier S. 134. 